\title{
In silico identification and characterization of the WRKY gene superfamily in pepper (Capsicum annuum L.)
}

Y. Cheng ${ }^{1}$, Z.P. Yao ${ }^{1}$, M.Y. Ruan', Q.J. Ye ${ }^{1}$, R.Q. Wang ${ }^{1}$, G.Z. Zhou ${ }^{1}$, J. Luo' ${ }^{2}$ Z.M. Li ${ }^{1}$, Y.J. Yang ${ }^{1}$ and H.J. Wan ${ }^{1}$

${ }^{1}$ State Key Laboratory Breeding Base for Zhejiang Sustainable Pest and Disease Control, Institute of Vegetables,

Zhejiang Academy of Agricultural Sciences, Hangzhou, China

${ }^{2}$ Institute of Digital Agriculture,

Zhejiang Academy of Agricultural Sciences, Hangzhou, Zhejiang, China

Corresponding authors: Y.J. Yang / H.J. Wan

E-mail: younghz@163.com / wanhongjian@sina.com

Genet. Mol. Res. 15 (3): gmr.15038675

Received March 30, 2016

Accepted July 14, 2016

Published September 23, 2016

DOI http://dx.doi.org/10.4238/gmr.15038675

Copyright (C) 2016 The Authors. This is an open-access article distributed under the terms of the Creative Commons Attribution ShareAlike (CC BY-SA) 4.0 License

\begin{abstract}
The WRKY family is one of the most important transcription factor families in plants, involved in the regulation of a broad range of biological roles. The recent releases of whole-genome sequences of pepper (Capsicum annuиm L.) allow us to perform a genome-wide identification and characterization of the WRKY family. In this study, 61 CaWRKY proteins were identified in the pepper genome. Based on protein structural and phylogenetic analyses, these proteins were classified into four main groups (I, II, III, and NG), and Group II was further divided into five subgroups (IIa to IIe). Chromosome mapping analysis indicated that CaWRKY genes are distributed across all 12 chromosomes, although the location of four
\end{abstract}


CaWRKYs (CaWRKY58-CaWRKY61) could not be identified. Two pairs of CaWRKYs located on chromosome 01 appear to be tandem duplications. Furthermore, the phylogenetic tree showed a close evolutionary relationship of WRKYs in three species from Solanaceae. In conclusion, this comprehensive analysis of CaWRKYs will provide rich resources for further functional studies in pepper.

Key words: WRKY; Transcription factors; Phylogenetic analysis; Pepper

\section{INTRODUCTION}

WRKY is one of the most important transcription factors in higher plants. As a sequence-specific DNA-binding transcription factor, the WRKY protein contains at least one WRKY domain, which is responsible for the special cis-element (w-box: TTGACC/T) binding activity. All WRKY domains contain a highly conserved peptide (WRKYGQK) at the N terminus, which is followed by a zinc-finger structure $(\mathrm{Cx} 4-5 \mathrm{Cx} 22-23 \mathrm{HxH}$ or $\mathrm{Cx} 7 \mathrm{Cx} 23 \mathrm{HxC}$; Rushton et al., 2010). The WRKY domain is assembled into a four-stranded $\beta$-sheet and a zinc-binding pocket for w-box binding and protein interaction activity. This could help regulate corresponding physiological processes by activating/repressing transcription of target genes (Yamasaki et al., 2005). It is well known that WRKY originated in lower eukaryotes gradually evolved into a large superfamily in higher plants (Rushton et al., 2010). Only one group of WRKY proteins (known as Group I) has been identified both in higher plants and in lower eukaryotes such as the nonphotosynthetic slime mold, Dictyostelium discoideum, and the green alga, Chlamydomonas reinhardtii. Thus, members of this group are believed to be most similar to the ancestral protein (Yamasaki et al., 2005; Rushton et al., 2010).

Based on the number of WRKY domains and the corresponding zinc-finger structure, WRKY proteins are classified mainly into three groups (I, II, and III; Huang et al., 2012). Group I contains two zinc-finger motifs (CX4CX22-23HXH), Group II contains one zincfinger motif (CX4CX22-23HxH), and Group III contains one zinc-finger motif (CX7CX2324HXC or CX4CX23HXC). Group II proteins can be further divided into five subgroups (IIa, IIb, IIc, IId, and IIe; Huang et al., 2012), and phylogenetic analyses have indicated an additional possible level of classification as IIa $+b$, IIc, and IId $+e$ (Zhang and Wang, 2005). Recently, Huang et al. (2012) found that among 81 tomato SIWRKYs, 15 were classified into Group I, 52 into Group II, and 11 into Group III. The remaining three SIWRKYs were placed in a new group labeled as none group (NG).

Since the first identification of plant WRKY proteins in sweet potato (Ishiguro and Nakamura, 1994; Rushton et al., 1996), many WRKYs from various plant species have been identified and characterized (Liu et al., 2014; Cai et al., 2015; Sun et al., 2015). Studies of WRKY transcription factors are no longer limited to model plants such as Arabidopsis and Oryza sativa (rice; Eulgem and Somssich, 2007; Mao et al., 2007). In members of Solanaceae, such as potato, eggplant, and tomato, WRKYs have been identified and characterized across the genome (Huang et al., 2012; Huang and Liu, 2013; Yang et al., 2015). Recently, LeWRKY was cloned in tomato and was induced by jasmonic acid and Botrytis cinerea, but not by salicylic acid (Lu et al., 2015).

Genetics and Molecular Research 15 (3): gmr.15038675 
In pepper (Capsicum annuum L.), the only proteins that have been reported to be involved in the regulation of various stress responses are several CaWRKYs and CaHsfA2 (Huh et al., 2012; Dang et al., 2014; Guo et al., 2014; Cai et al., 2015). Prior to this study, pepper CaWRKY had not been comprehensively analyzed at the whole-genome level. Recently, whole-genome sequencing for pepper has provided an opportunity for comprehensive identification and analysis of CaWRKY transcription factors (Qin et al., 2014). In the current study, we identified a total of 61 CaWRKYs based on the Pepper Genome Database (PGD; http://peppersequence.genomics.cn; Qin et al., 2014). Further bioinformatic analyses included comparisons of conserved domains, assessment of gene structure and classification, mapping onto chromosomes, and phylogenetic inference. Our results will provide resources for future studies on the functions of CaWRKY genes.

\section{MATERIAL AND METHODS}

\section{Identification and intron-exon configuration of CaWRKYs in pepper}

The annotated protein sequences of Capsicum annuиm L. 'Zunla-1' were downloaded from the PGD (Release 2.0, http://peppersequence.genomics.cn; Qin et al., 2014). A BLASTp was performed using the HMM profile (Pfam: PF03106) of the conserved WRKY domain (http://pfam.xfam.org/). Default parameters were employed and the e-value was set at $1 \mathrm{e}^{-5}$. The full set of non-redundant putative CaWRKYs was then further checked for the presence of WRKY domains using Pfam 27.0 (Punta et al., 2012). Genomic DNA sequences, including the encoding sequences of CaWRKYs were also downloaded from the PGD. The intron-exon structure was visualized using GSDS 2.0 (http://gsds.cbi.pku.edu.cn).

\section{Multiple-sequence alignment and chromosome mapping of CaWRKYs}

To reveal the level of sequence conservation of these CaWRKY proteins, the sequences of the specific WRKY domain(s) of each CaWRKY were detected using SMART7.0 (http:// smart.embl-heidelberg.de/; Letunic et al., 2012). Preliminary sequence manipulations were performed using DNAMAN 6.0. Multiple-sequence alignment of CaWRKY domains was conducted using BioEdit 7.0.

Chromosome mapping of each CaWRKY was performed with MapDrawV2.1 based on gene information provided in the PGD. Tandem duplications of CaWRKY genes were identified using the method of Yang et al. (2008) and Huang et al. (2012). Genes were identified as tandem duplicates if they were found within $100 \mathrm{~kb}$ of each other, using Smith-Waterman alignment (e-values $\leq 1 \times 10^{-5}$ ).

\section{Phylogenetic analysis of CaWRKYs}

Amino acid sequences of all CaWRKYs were aligned using ClustalX version 1.83 with default settings (http://www.clustal.org/; Thompson et al., 1997). A phylogenetic analysis of the WRKY conserved domains was conducted in MEGA version 5.05 (Tamura et al., 2011). Relative branch support was evaluated using bootstraps (1000 replicates). Branch lengths were calculated by pairwise comparison of genetic distances, and missing data were treated by pairwise deletions of gaps. 


\section{RESULTS}

\section{Identification of CaWRKY proteins from the pepper genome}

A total of 61 CaWRKYs in C. annuum L. 'Zunla-1' were identified and chosen for analysis. These proteins were named CaWRKY01 to CaWRKY61 (Table 1). Among the 61, CaWRKY33 is the longest protein ( 869 aa), CaWRKY58 is the shortest (137aa), and the average length is 358 bp. Detailed information, including accession number, WRKYGQK peptide, zinc-finger structure, WRKY domain number, and group classification are listed in Table 1 and Table S1.

\begin{tabular}{|c|c|c|c|c|c|}
\hline Gene symbol & Gene locus & $\begin{array}{c}\text { WRKY domain } \\
\text { Conserved heptapeptide }\end{array}$ & Zinc-finger type & Domain number & Group \\
\hline CaWRKY01 & Capana01g000165 & WRKYGQK & Deficiency & 1 & NG \\
\hline CaWRKY02 & Capana01g000167 & WRKYGQK & $\mathrm{C} 2 \mathrm{H} 2$ & 1 & $\mathrm{IIe}$ \\
\hline CaWRKY03 & $\begin{array}{l}\text { Capana01g002803 } \\
\end{array}$ & WRKYGQK & $\mathrm{C} 2 \mathrm{H} 2$ & 1 & III \\
\hline CaWRKY04 & Capana01g003441 & WRKYGQK & $\mathrm{C} 2 \mathrm{H} 2$ & 1 & IIc \\
\hline CaWRKY05 & Capana01g004471 & WRKYGQK & $\mathrm{C} 2 \mathrm{HC}$ & 1 & III \\
\hline CaWRKY06 & $\begin{array}{l}\text { Capana01g004472 } \\
\end{array}$ & WRKYGQK & $\mathrm{C} 2 \mathrm{HC}$ & 1 & IIII \\
\hline CaWRKY07 & Capana02g000212 & WRKYGQK & $\mathrm{C} 2 \mathrm{H} 2$ & 1 & $\mathrm{IIe}$ \\
\hline CaWRKY08 & Capana02g000680 & WRKYGQK & $\mathrm{C} 2 \mathrm{H} 2$ & 1 & IId \\
\hline CaWRKY09 & $\begin{array}{l}\text { Capana02g000918 } \\
\end{array}$ & WRKYGQK & $\mathrm{C} 2 \mathrm{H} 2$ & 1 & $\mathrm{IIb}$ \\
\hline CaWRKY10 & $\begin{array}{l}\text { Capana02g001642 } \\
\end{array}$ & WRKYGQK & $\mathrm{C} 2 \mathrm{H} 2$ & 1 & IIIe \\
\hline CaWRKY11 & Capana02g002230 & WRKYGQK & $\mathrm{C} 2 \mathrm{H} 2$ & 1 & $\mathrm{IIb}$ \\
\hline CaWRKY12 & Capana02g003053 & WRKYGQK & Deficiency & 1 & NG \\
\hline CaWRKY13 & Capana02g003339 & WRKYGQK/WRKYGQK & $\mathrm{C} 2 \mathrm{H} 2$ & 1 & I \\
\hline CaWRKY14 & Capana02g003661 & WRKYGQK & $\mathrm{C} 2 \mathrm{H} 2$ & 2 & IIc \\
\hline CaWRKY15 & Capana03g000473 & WRKYGQK & $\mathrm{C} 2 \mathrm{H} 2$ & 1 & IIa \\
\hline CaWRKY16 & Capana03g001099 & WRKYGQK & $\mathrm{C} 2 \mathrm{H} 2$ & 1 & $\mathrm{IIb}$ \\
\hline CaWRKY17 & Capana03g001962 & WRKYGMK & $\mathrm{C} 2 \mathrm{H} 2$ & 1 & NG \\
\hline CaWRKY18 & Capana03g002072 & WRKYGQK & $\mathrm{C} 2 \mathrm{H} 2$ & 1 & IIII \\
\hline CaWRKY19 & Capana03g002134 & WRKYGQK & $\mathrm{C} 2 \mathrm{H} 2$ & 1 & NG \\
\hline CaWRKY20 & Capana03g002635 & WRKYGQK & $\mathrm{C} 2 \mathrm{HC}$ & 1 & III \\
\hline CaWRKY21 & $\begin{array}{l}\text { Capana03g003085 } \\
\end{array}$ & WRKYGQK/WRKYGQK & $\mathrm{C} 2 \mathrm{H} 2$ & 2 & I \\
\hline CaWRKY22 & $\begin{array}{l}\text { Capana03g003279 } \\
\end{array}$ & WRKYGQK & $\mathrm{C} 2 \mathrm{H} 2$ & 1 & IId \\
\hline CaWRKY23 & Capana04g000568 & WRKYGQK & $\mathrm{C} 2 \mathrm{H} 2$ & 1 & IId \\
\hline CaWRKY24 & Capana04g001820 & WRKYGQK/WRKYGQK & $\mathrm{C} 2 \mathrm{H} 2$ & 2 & I \\
\hline CaWRKY25 & Capana05g002502 & WRKYGQK/WRKYGQK & $\mathrm{C} 2 \mathrm{H} 2$ & 2 & I \\
\hline CaWRKY26 & $\begin{array}{l}\text { Capana06g001008 } \\
\end{array}$ & WRKYGQK & $\mathrm{C} 2 \mathrm{H} 2$ & 1 & $\mathrm{Ilb}$ \\
\hline CaWRKY27 & Capana06g001110 & WRKYGQK & $\mathrm{C} 2 \mathrm{H} 2$ & 1 & IIa \\
\hline CaWRKY28 & Capana06g001506 & WRKYGQK/WRKYGQK & $\mathrm{C}_{2} \mathrm{H} 2$ & 2 & I \\
\hline CaWRKY29 & $\begin{array}{l}\text { Capana06g002128 } \\
\end{array}$ & WRKYGQK & $\mathrm{C} 2 \mathrm{HC}$ & 1 & IIII \\
\hline CaWRKY30 & Capana06g003072 & WRKYGQK & $\mathrm{C} 2 \mathrm{H} 2$ & 1 & IId \\
\hline CaWRKY31 & $\begin{array}{l}\text { Capana07g000181 } \\
\end{array}$ & WRKYGQK/WRKYGQK & $\mathrm{C} 2 \mathrm{H} 2$ & 2 & I \\
\hline CaWRKY32 & Capana07g000528 & WRKYGQK & $\mathrm{C} 2 \mathrm{H} 2$ & 1 & III \\
\hline CaWRKY33 & Capana07g001256 & WRKYGQK/WRKYGQK & $\mathrm{C} 2 \mathrm{H} 2$ & 2 & I \\
\hline CaWRKY34 & Capana07g001387 & WRKYGQK & $\mathrm{C} 2 \mathrm{H} 2$ & 1 & $\mathrm{IIb}$ \\
\hline CaWRKY35 & Capana07g001809 & WRKYGQK & $\mathrm{C} 2 \mathrm{H} 2$ & 1 & IIe \\
\hline CaWRKY36 & Capana07g001968 & WRKYGQK & $\mathrm{C} 2 \mathrm{H} 2$ & 1 & IIc \\
\hline CaWRKY37 & Capana07g002350 & WRKYGQK/WRKYGQK & $\mathrm{C} 2 \mathrm{H} 2$ & 2 & I \\
\hline CaWRKY38 & $\begin{array}{l}\text { Capana07g002454 } \\
\end{array}$ & WRKYGQK/WRKYGQK & $\mathrm{C} 2 \mathrm{H} 2$ & 2 & I \\
\hline CaWRKY39 & Capana08g000429 & WRKYGKK & $\mathrm{C} 2 \mathrm{H} 2$ & 1 & IIc \\
\hline CaWRKY40 & Capana08g000683 & WRKYGQK & $\mathrm{C} 2 \mathrm{H} 2$ & 1 & IIa \\
\hline CaWRKY41 & Capana08g001012 & WRKYGQK & $\mathrm{C} 2 \mathrm{H} 2$ & 1 & IIe \\
\hline CaWRKY42 & Capana08g001044 & WRKYGQK & $\mathrm{C} 2 \mathrm{HC}$ & 1 & III \\
\hline CaWRKY43 & Capana08g001961 & WRKYGQK & $\mathrm{C} 2 \mathrm{H} 2$ & 1 & $\mathrm{IIb}$ \\
\hline CaWRKY44 & $\begin{array}{l}\text { Capana09g000676 } \\
\end{array}$ & WRKYGQK & $\mathrm{C} 2 \mathrm{H} 2$ & 1 & III \\
\hline CaWRKY45 & Capana09g001251 & WRKYGQK/WRKYGQK & $\mathrm{C} 2 \mathrm{H} 2$ & 2 & I \\
\hline CaWRKY46 & Capana09g001790 & WRKYGQK & $\mathrm{C} 2 \mathrm{HC}$ & 1 & NG \\
\hline CaWRKY47 & Capana10g000205 & WRKYGQN/no conserved stretch & $\mathrm{C} 2 \mathrm{H} 2$ & 2 & I \\
\hline CaWRKY48 & Capana10g000754 & WRKYGQK & $\mathrm{C} 2 \mathrm{H} 2$ & 1 & IIe \\
\hline CaWRKY49 & Capana10g001220 & WRKYGQK & $\mathrm{C} 2 \mathrm{HC}$ & 1 & III \\
\hline CaWRKY50 & $\begin{array}{l}\text { Capana10g001548 } \\
\end{array}$ & WRKYGQK & $\mathrm{C} 2 \mathrm{HC}$ & 1 & III \\
\hline CaWRKY51 & Capana10g001791 & WRKYGQK/WRKYGHK & $\mathrm{C} 2 \mathrm{H} 2$ & 2 & I \\
\hline CaWRKY52 & Capana10g001805 & WRKYGQK & $\mathrm{C} 2 \mathrm{H} 2$ & 1 & NG \\
\hline CaWRKY53 & $\begin{array}{l}\text { Capana1lg001882 } \\
\end{array}$ & WRKYGQK/WRKYGQK & $\mathrm{C} 2 \mathrm{H} 2$ & 2 & I \\
\hline CaWRKY54 & Capana11g001905 & WRKYGQK & $\mathrm{C} 2 \mathrm{H} 2$ & 1 & IIIc \\
\hline CaWRKY55 & Capana12g001134 & WRKYGQK & $\mathrm{C} 2 \mathrm{H} 2$ & 1 & IIa \\
\hline CaWRKY56 & $\begin{array}{l}\text { Capana12g001826 } \\
\end{array}$ & WRKYGKK & $\mathrm{C}_{2} \mathrm{H}_{2}$ & 1 & IIIc \\
\hline CaWRKY57 & Capana12g001851 & WRKYGQK & $\mathrm{C} 2 \mathrm{H} 2$ & 1 & IIIc \\
\hline CaWRKY58 & Capana00g000429 & KKKGEK & $\mathrm{C} 2 \mathrm{H} 2$ & 1 & IIc \\
\hline CaWRKY59 & Capana00g001033 & WRKYGQK & $\mathrm{C} 2 \mathrm{H} 2$ & 1 & IIc \\
\hline CaWRKY60 & Capana00g003083 & WRKYGQK & $\mathrm{C} 2 \mathrm{H} 2$ & 1 & IId \\
\hline CaWRKY61 & Capana00g004112 & WRKYGKK & $\mathrm{C} 2 \mathrm{H} 2$ & 1 & IIc \\
\hline
\end{tabular}

NG means none group. CaWRKY01, CaWRKY12, CaWRKY17, CaWRKY19, CaWRKY46, and CaWRKY52 were not assigned to any group. The variants of conserved WRKYGQK peptide are shown in bold and no conserved WRKYGQK stretch exists in the C-terminal of CaWRKY47.

Genetics and Molecular Research 15 (3): gmr.15038675 


\section{Classification and structural analysis of $C a W R K Y$ genes}

As the most prominent structural feature of WRKY transcription factors, a total of 74 WRKY domains were identified in the 61 CaWRKY proteins. When there were two domains in the same WRKY protein, we designated them as the N-terminal WRKY domain (I-NTWD) and the C-terminal WRKY domain (I-CTWD).

CaWRKY01 and CaWRKY12 encoded truncated proteins, and they were excluded from the alignment of CaWRKY domains in ClustalX. The results classified the CaWRKYs into four groups (I, II, III, and NG). Thirteen CaWRKYs were classified into Group I, and they contained two WRKY domains with the $\mathrm{C} 2 \mathrm{H} 2$ type of zinc finger structure (CX4CX22-23HX1H). Group II comprised $33 \mathrm{CaWRKYs}$ that each contained only one WRKY domain with the $\mathrm{C} 2 \mathrm{H} 2$ type of zinc-finger (CX4-5CX23HX1H). These were further divided into five subgroups: IIa (4 proteins), IIb (6), IIc (12), IId (5), and IIe (6). Group III contained nine CaWRKY members that also had one WRKY domain, but the zinc-finger (C2HC: CX7CX23-24HX1H/CX4CX23HX1H) was different in the other two groups. The remaining four CaWRKYs (CaWRKY17, CaWRKY19, CaWRKY46, and CaWRKY52) did not seem to fit into any of the groups above because of their distinctive WRKY domain structure and were classified in NG. The detailed amino acid sequences of the 74 CaWRKY domains are in Table S2.

Although the WRKYGQK peptide is known to be highly conserved, sequence variation was still found in six of these CaWRKYs (Table 1). In addition to the most common variant (WRKYGKK), three other variants (WRKYGMK, WRKYGQN, and WRKYGHK) were also identified in our study. Strikingly, a new variant (KKKGEK) was observed for the CaWRKY58 protein and a WRKY domain deletion event had occurred in the C-terminal WRKY domain of CaWRKY47. In addition, the expected variant, C-X33, was identified in the zinc-finger structure of CaWRKY32 (Table 1).

Most CaWRKY members have 2-3 introns, except for those in Group I and Group IIb, in which most members seem to have at least 4 introns (Figure 1). CaWRKY33 in Group I has the most introns (12), while several CaWRKYs have only one intron (CaWRKY14 and 58 in Group IIc, CaWRKY06 in Group III; Figure 1).

\section{Multiple-sequence alignment of the WRKY gene superfamily}

As shown in Figure 2, most of the WRKYGQK variants were members of Group IIc, including WRKYGKKs in CaWRKY39, CaWRKY56, and CaWRKY61, and KKKGEK in CaWRKY58. The other variants were in Group I and NG. By analyzing the WRKY domain sequence of CaWRKY members in the NG, we found that some of the more unusual variations were observed in the WRKY domains of CaWRKY17, CaWRKY19, and CaWRKY46, compared to members of Groups I, II, and III. For example, the amino acid residue immediately after the first Cys (C) of the zinc-finger in CaWRKY17 and CaWRKY19 is Asn (N), instead of T, D, or S. For CaWRKY46, both the amino acids adjacent to the second His $(\mathrm{H})$ of the zincfinger are Leu (L) (Figure 2).

\section{Chromosomal distribution and duplication of $C a W R K Y$ genes}

The genomic locations of 57 of the 61 identified CaWRKY genes across the twelve pepper chromosomes. The remaining four genes (CaWRKY58 to CaWRKY61) could not be 


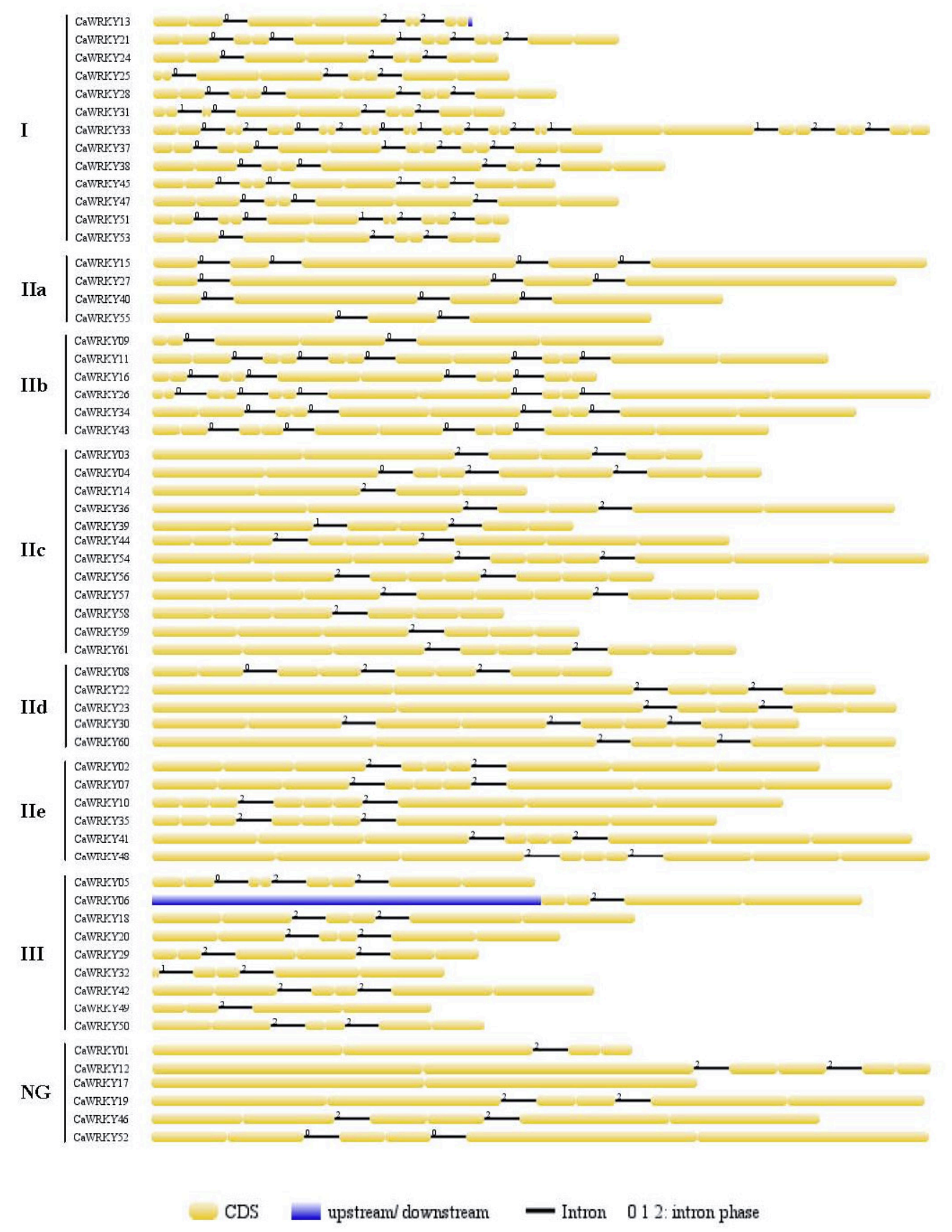

Figure 1. Intron-extron structure of CaWRKY genes in pepper. The upstream/downstream, extron and intron are shown with blue rectangle, yellow cylinder and black line, respectively. Intron phase numbers $(0,1,2)$ were labeled on the top of each CaWRKY gene sketch map.

Genetics and Molecular Research 15 (3): gmr.15038675 


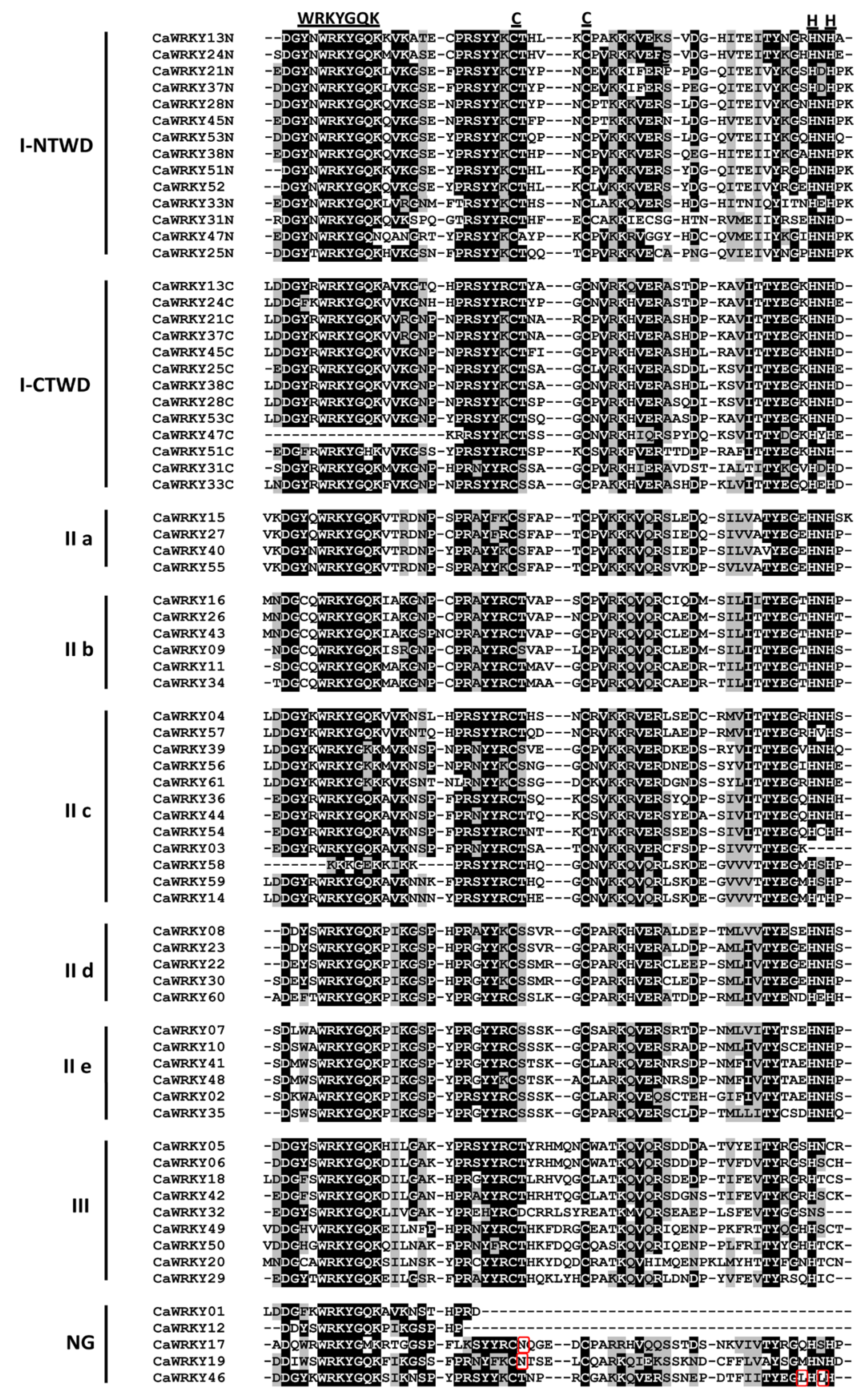

Figure 2. Multiple-sequence alignment of conserved WRKY domains of different groups in pepper. CaWRKY domains that belong to the same group are clustered together. The conserved amino acids are emphasized with black background. The conserved heptapeptide (WRKYGQK) and zinc-finger key structure [cysteine (C), histidine $(\mathrm{H})]$ are labeled on top. The amino acid variations of NG group of members are labeled with red blocks. 
located on any chromosome and were assigned to chromosome 00. Although the sequenced size of chromosome $02(164.0 \mathrm{M})$ only accounts for $5 \%$ of the assembled pepper genome (3.36 G), it includes 8 of 61 CaWRKYs ( 13\%). In contrast, chromosome $05(217.27 \mathrm{M})$ only contains one, CaWRKY25. Overall, more CaWRKY genes are found on chromosomes 01, 02, 03, 06, 07, 08, and 10 than on chromosomes 04, 05, 09, 11, and 12 (Figure 3).

Two pairs of tandem duplications of CaWRKY genes were found. A pair of homologous genes was considered a tandem duplication if the genes were within $100 \mathrm{~kb}$ of each other. As shown in Figure 3, these pairs of genes (CaWRKY01/CaWRKY02, CaWRKY05/CaWRKY06) were both located on chromosome 01 .

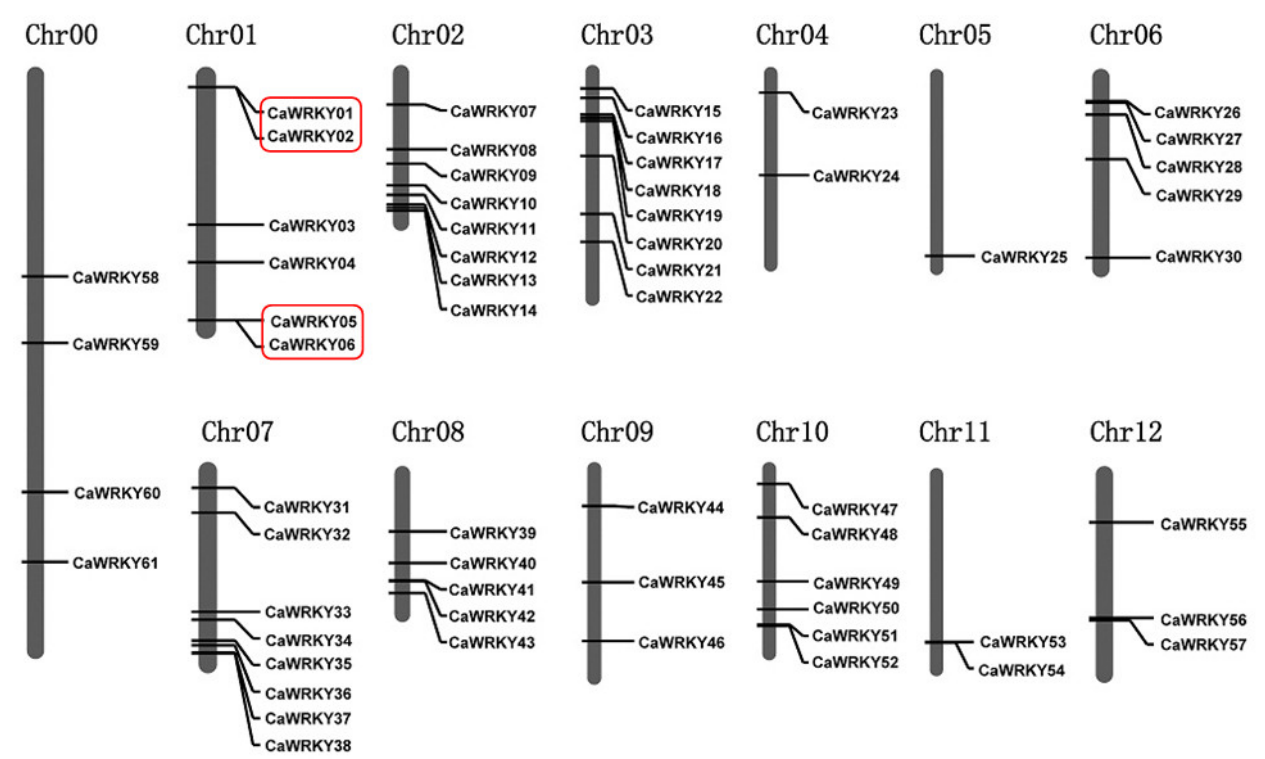

Figure 3. Chromosome distribution of CaWRKY genes. Tandemly duplication genes are labeled with red rectangles.

\section{Phylogenetic analysis of the WRKY gene family in pepper, tomato, and potato}

We inferred unrooted phylogenetic relationships among the CaWRKY proteins in pepper, using an alignment of amino acid sequences of 72 WRKY domains (including N-terminal and C-terminal domains of Group I members; Figure 4). Eight at WRKYs from Arabidopsis were also selected for phylogenetic analysis. The relationships of CaWRKYs in the tree confirm the groupings noted above (Figure 4 and Table 1). Three main clusters were observed, cluster 1 (Group I, IIa+b, and IIc), cluster 2 (Group IId and IIe), and cluster 3 (Group III; Figure 4). The most strongly supported clusters and subclusters are I+IIc and IIa $+\mathrm{b}$ (in cluster 1) and cluster $2(\mathrm{IId}+\mathrm{e})$. Interestingly, independent distinct gene expansion events occurred in cluster 2 .

To further illuminate the evolutionary relationships among WRKY genes in Solanaceae, we ran a separate phylogenetic analysis including amino acid sequences of 264 WRKY domains (including N-terminal and C-terminal domains of members of Group I) from pepper, tomato, and potato (Figure 5). Incomplete WRKY domains (CaWRKY01 and CaWRKY12 in pepper, and StWRKY21 and StWRKY39C in potato) were excluded from 


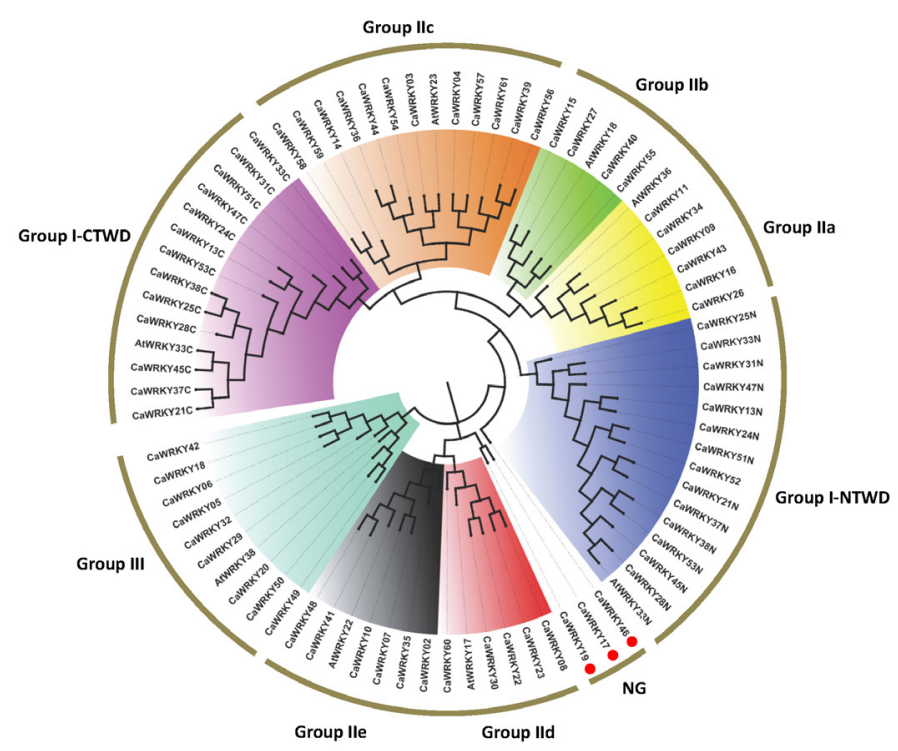

Figure 4. Phylogenetic tree of CaWRKY proteins in pepper using the neighbor joining method by MEGA 5.0. The WRKY domains of each group are clustered together, and each clade was labeled with different colors. The three CaWRKYs in no-color clade (CaWRKY17, CaWRKY19 and CaWRKY46) were defined as none group (NG) and emphasized with red spots.

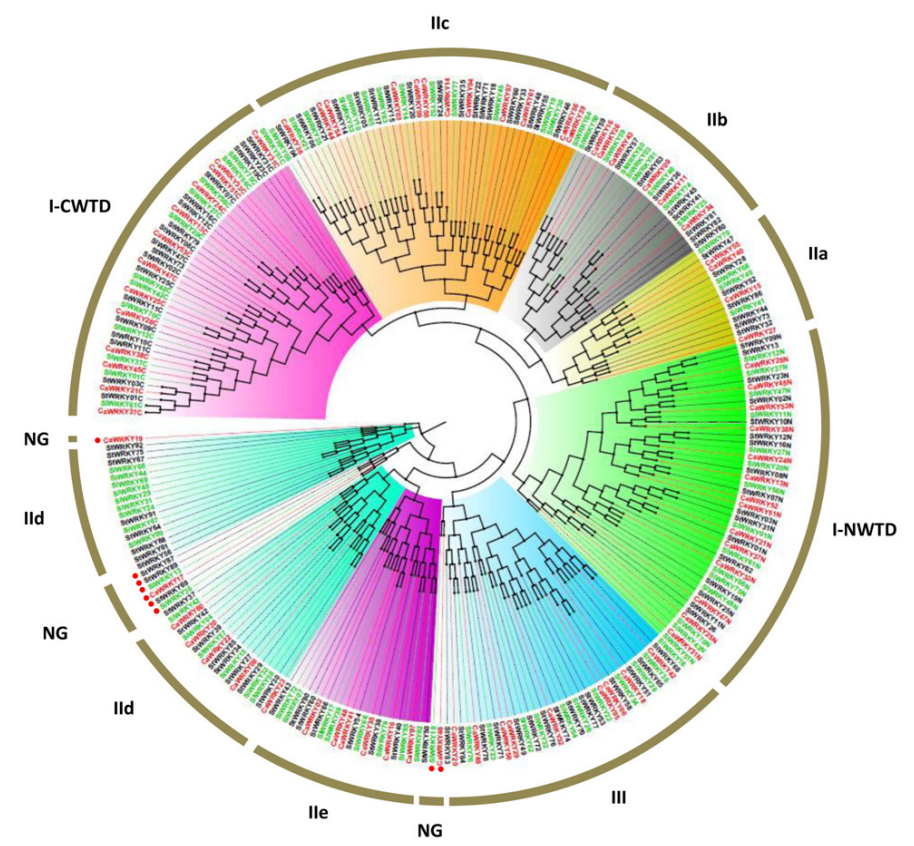

Figure 5. Phylogenetic tree of WRKY proteins among pepper, tomato and potato using the neighbor joining method by MEGA 5.0. Each WRKY group is labeled with different colors. None group (NG) WRKYs are emphasized with red spots.

Genetics and Molecular Research 15 (3): gmr.15038675 
the phylogenetic analysis. The amino acid sequences of all 268 WRKY domains are available in Table S2. The resulting topology of this tree is completely consistent with the CaWRKY phylogenetic tree (Figure 4), but reveals a more complex set of relationships (Figure 5). The interspersed distribution of WRKY domains from the three species (pepper, tomato, and potato) in all of the groups suggests that the expansion of WRKY genes occurred in an ancestor of these three species. In addition, WRKY gene expansions were observed for nine members (CaWRKY17, CaWRKY19, CaWRKY46, StWRKY69, StWRKY87, StWRKY89, SIWRKY13, SIWRKY18, and SIWRKY36; Figure 5), suggesting the evolutionary uniqueness of these WRKY members in the long-term evolution of this gene family.

\section{DISCUSSION}

The WRKY transcription factor family has been noted in many plant species for its regulatory roles in a wide range of biological processes. Seven WRKY protein-encoding genes from pepper had been reported to play critical roles in biological processes, including pathogen resistance and high-temperature tolerance (Park et al., 2006; Lim et al., 2011; Wang and He, 2011; Huh et al., 2012; Wang et al., 2013; Dang et al., 2013, 2014; Cai et al., 2015). Based on the recently released pepper genome sequence of $C$. annuum 'Zunla-1', we identified the entire family of $61 \mathrm{CaWRKY}$ proteins in pepper. These CaWRKYs are classified into Group I, Group II (IIa, IIb, IIc, IId, and IIe), Group III, and NG, which possess 21.3, 54.1\% (6.6, 9.8, 19.7, 8.2, and 9.8\%), 14.8, and 9.8\% of all CaWRKYs, respectively (Table 1 and Table S2).

Gene duplication is an important factor in the evolution of gene families. In our study, about 7\% (4 of 61) CaWRKY genes were shown to result from tandem duplication (Figure 3). The different numbers of introns of CaWRKY genes in different groups suggest that some of the loss/gain events occurred prior to the speciation of Capsicum (Figure 1). A phylogenetic tree of WRKYs from three species in Solanaceae (pepper, tomato, and potato) confirmed that members of Group II can be reorganized into three clusters, including Group IIa+b, IIc, and IId+e (Figures 4, 5). A unique gene expansion within Group IId+e had been identified in tomato (Huang et al., 2012) and potato (Huang and Liu, 2013). In the present study, nine WRKYs (three CaWRKYs, three SIWRKYs, and three StWRKYs) were shown to form a separate subclade in GroupIId+e, demonstrating that a unique WRKY gene expansion has occurred in Solanaceae (Figures 4 and 5). We propose that these WRKYs play specific roles in Solanaceae plants.

In this study, genome-wide identification and analysis of the CaWRKY gene superfamily in pepper were performed using bioinformatic methods. The results showed at least 61 members of the family across the genome. In addition, studies of exon-intron structure, multiple-sequence alignment, chromosome mapping, and phylogenetic analyses of these 61 CaWRKYs were also conducted. The results provide important insights for future studies of the structure, evolution, and biological functions of this critical transcription factor family in pepper.

\section{Conflicts of interest}

The authors declare no conflict of interest.

\section{ACKNOWLEDGMENTS}

Research supported by the Zhejiang Provincial Natural Science Foundation of China

Genetics and Molecular Research 15 (3): gmr.15038675 
(\#Q15C150010), the National Natural Science Foundation of China (\#31301774, \#31272156, and \#3150110311), the Young Talent Cultivation Project of Zhejiang Academy of Agricultural Sciences (\#2015R23R08E07, \#2015R23R08E09), the Public Agricultural Technology Research in Zhejiang (\#2016C32101, \#2015C32049), and the Technological System of Ordinary Vegetable Industry (\#CARS-25-G-16).

\section{REFERENCES}

Cai H, Yang S, Yan Y, Xiao Z, et al. (2015). CaWRKY6 transcriptionally activates CaWRKY40, regulates Ralstonia solanacearum resistance, and confers high-temperature and high-humidity tolerance in pepper. J. Exp. Bot. 66: 31633174. http://dx.doi.org/10.1093/jxb/erv125

Dang F, Wang Y, She J, Lei Y, et al. (2014). Overexpression of CaWRKY27, a subgroup IIe WRKY transcription factor of Capsicum annuum, positively regulates tobacco resistance to Ralstonia solanacearum infection. Physiol. Plant. 150: 397-411. http://dx.doi.org/10.1111/ppl.12093

Dang FF, Wang YN, Yu L, Eulgem T, et al. (2013). CaWRKY40, a WRKY protein of pepper, plays an important role in the regulation of tolerance to heat stress and resistance to Ralstonia solanacearum infection. Plant Cell Environ. 36: 757-774. http://dx.doi.org/10.1111/pce.12011

Eulgem T and Somssich IE (2007). Networks of WRKY transcription factors in defense signaling. Curr. Opin. Plant Biol. 10: 366-371. http://dx.doi.org/10.1016/j.pbi.2007.04.020

Guo M, Yin YX, Ji JJ, Ma BP, et al. (2014). Cloning and expression analysis of heat-shock transcription factor gene CaHsfA2 from pepper (Capsicum annuum L.). Genet. Mol. Res. 13: 1865-1875. http://dx.doi.org/10.4238/2014. March.17.14

Huang S, Gao Y, Liu J, Peng X, et al. (2012). Genome-wide analysis of WRKY transcription factors in Solanum lycopersicum. Mol. Genet. Genomics 287: 495-513. http://dx.doi.org/10.1007/s00438-012-0696-6

Huang SX and Liu YS (2013). The bioinformatics analysis of WRKY transcription factors in potato. Chin. J. Appl. Environ. Biol. 19: 205-214. http://dx.doi.org/10.3724/SP.J.1145.2013.00205

Huh SU, Choi LM, Lee GJ, Kim YJ, et al. (2012). Capsicum annuum WRKY transcription factor d (CaWRKYd) regulates hypersensitive response and defense response upon Tobacco mosaic virus infection. Plant Sci. 197: 50-58. http:// dx.doi.org/10.1016/j.plantsci.2012.08.013

Ishiguro S and Nakamura K (1994). Characterization of a cDNA encoding a novel DNA-binding protein, SPF1, that recognizes SP8 sequences in the 5' upstream regions of genes coding for sporamin and $\beta$-amylase from sweet potato. Mol. Gen. Genet. 244: 563-571. http://dx.doi.org/10.1007/BF00282746

Letunic I, Doerks T and Bork P (2012). SMART 7: recent updates to the protein domain annotation resource. Nucleic Acids Res. 40: D302-D305. http://dx.doi.org/10.1093/nar/gkr931

Lim JH, Park CJ, Huh SU, Choi LM, et al. (2011). Capsicum annuum WRKYb transcription factor that binds to the CaPR-10 promoter functions as a positive regulator in innate immunity upon TMV infection. Biochem. Biophys. Res. Commun. 411: 613-619. http://dx.doi.org/10.1016/j.bbrc.2011.07.002

Liu B, Hong YB, Zhang YF, Li XH, et al. (2014). Tomato WRKY transcriptional factor SIDRW1 is required for disease resistance against Botrytis cinerea and tolerance to oxidative stress. Plant Sci. 227: 145-156. http://dx.doi. org/10.1016/j.plantsci.2014.08.001

Lu M, Wang LF, Du XH, Yu YK, et al. (2015). Molecular cloning and expression analysis of jasmonic acid dependent but salicylic acid independent LeWRKY1. Genet. Mol. Res. 14: 15390-15398. http://dx.doi.org/10.4238/2015. November. 30.16

Mao P, Duan M, Wei C and Li Y (2007). WRKY62 transcription factor acts downstream of cytosolic NPR1 and negatively regulates jasmonate-responsive gene expression. Plant Cell Physiol. 48: 833-842. http://dx.doi.org/10.1093/pcp/ pcm058

Park CJ, Shin YC, Lee BJ, Kim KJ, et al. (2006). A hot pepper gene encoding WRKY transcription factor is induced during hypersensitive response to tobacco mosaic virus and Xanthomonas campestris. Planta 223: 168-179.

Punta M, Coggill PC, Eberhardt RY, Mistry J, et al. (2012). The Pfam protein families database. Nucleic Acids Res. 40: D290-D301. http://dx.doi.org/10.1093/nar/gkr1065

Qin C, Yu C, Shen Y, Fang X, et al. (2014). Whole-genome sequencing of cultivated and wild peppers provides insights into Capsicum domestication and specialization. Proc. Natl. Acad. Sci. USA 111: 5135-5140. http://dx.doi.org/10.1073/ pnas. 1400975111

Rushton PJ, Torres JT, Parniske M, Wernert P, et al. (1996). Interaction of elicitor-induced DNA-binding proteins with

Genetics and Molecular Research 15 (3): gmr.15038675 
elicitor response elements in the promoters of parsley PR1 genes. EMBO J. 15: 5690-5700.

Rushton PJ, Somssich IE, Ringler P and Shen QJ (2010). WRKY transcription factors. Trends Plant Sci. 15: 247-258. http://dx.doi.org/10.1016/j.tplants.2010.02.006

Sun XC, Gao YF, Li HR, Yang SZ, et al. (2015). Over-expression of SlWRKY39 leads to enhanced resistance to multiple stress factors in tomato. J. Plant Biol. 58: 52-60. http://dx.doi.org/10.1007/s12374-014-0407-4

Tamura K, Peterson D, Peterson N, Stecher G, et al. (2011). MEGA5: molecular evolutionary genetics analysis using maximum likelihood, evolutionary distance, and maximum parsimony methods. Mol. Biol. Evol. 28: 2731-2739. http://dx.doi.org/10.1093/molbev/msr121

Thompson JD, Gibson TJ, Plewniak F, Jeanmougin F, et al. (1997). The CLUSTAL_X windows interface: flexible strategies for multiple sequence alignment aided by quality analysis tools. Nucleic Acids Res. 25: 4876-4882. http:// dx.doi.org/10.1093/nar/25.24.4876

Wang Y and He S (2011). Functional analysis of a new WRKY gene isolated from pepper (Capsicum annuum)[C]. International Conference on Remote Sensing, Environment and Transportation Engineering. IEEE, Nanjing, 68606864 .

Wang Y, Dang F, Liu Z, Wang X, et al. (2013). CaWRKY58, encoding a group I WRKY transcription factor of Capsicum annuum, negatively regulates resistance to Ralstonia solanacearum infection. Mol. Plant Pathol. 14: 131-144. http:// dx.doi.org/10.1111/j.1364-3703.2012.00836.x

Yamasaki K, Kigawa T, Inoue M, Tateno M, et al. (2005). Solution structure of an Arabidopsis WRKY DNA binding domain. Plant Cell 17: 944-956. http://dx.doi.org/10.1105/tpc.104.026435

Yang X, Kalluri UC, Jawdy S, Gunter LE, et al. (2008). The F-box gene family is expanded in herbaceous annual plants relative to woody perennial plants. Plant Physiol. 148: 1189-1200. http://dx.doi.org/10.1104/pp.108.121921

Yang X, Deng C, Zhang Y, Cheng Y, et al. (2015). The WRKY transcription factor genes in eggplant (Solanum melongena L.) and Turkey Berry (Solanum torvum Sw.). Int. J. Mol. Sci. 16: 7608-7626. http://dx.doi.org/10.3390/ijms16047608

Zhang Y and Wang L (2005). The WRKY transcription factor superfamily: its origin in eukaryotes and expansion in plants. BMC Evol. Biol. 5: 1. http://dx.doi.org/10.1186/1471-2148-5-1

\section{Supplementary material}

Table S1. CaWRKY gene family.

Table S2. WRKY domain of the WRKY gene superfamily in Solanaceae plants. 